\title{
COMPARACIÓN DE TRES AGENTES REMINERALIZANTES UTILIZADOS EN LESIONES DE MANCHAS BLANCAS EN PREMOLARES MEDIDOS CON FLUORESCENCIA LÁSER: UN ESTUDIO IN VITRO
}

\author{
COMPARISON OF THREE REMINERALIZATION AGENTS FOR WHITE SPOT LESIONS IN \\ PREMOLARS MEASURED BY LASER-INDUCED FLUORESCENCE: AN IN VITRO STUDY
}

María Alejandra Romero González ${ }^{1}$

marialeromer092@gmail.com

ORCID: 0000-0003-0123-1735
Denisse Pilar Carmen Aguilar Gálvez ${ }^{1}$

daguilar@cientifica.edu.pe

ORCID: 0000-0002-8394-7173

\section{RESUMEN}

Objetivo: Evaluar la remineralización de lesiones de manchas blancas en el esmalte de premolares humanos a través de la fluorescencia láser utilizando el barniz de flúor al 5\% (Duraphat $\left.{ }^{\circledR}\right)$, la nano-hidroxiapatita (Nano $\mathrm{P}^{\circledR}$ ), y la combinación de ambos agentes, a los 30 días de su aplicación. Método: La muestra estuvo conformada por 40 premolares y dividida en 4 grupos, (1) control (sin agente): saliva artificial, (2) barniz de flúor al 5\% (Duraphat $\left.{ }^{\circledR}\right)$, (3) nano-hidroxiapatita (Nano $\left.\mathrm{P}^{\circledR}\right)$, (4) y una combinación de ambos agentes remineralizantes (nanohidroxiapatita - Nano $\mathrm{P}^{\circledR}$ y barniz de flúor al $5 \%$ - Duraphat ${ }^{\circledR}$ ). Se analizaron los datos mediante la prueba de Anova de una vía y test de Bonferroni. Se trabajó con un nivel de significancia $p<0,05$. Resultados: La aplicación del barniz de flúor al $5 \%$ (Duraphat ${ }^{\circledR}$ ) y la nano-hidroxiapatita $\left(\right.$ Nano $\left.\mathrm{P}^{\circledR}\right)$, seguido del barniz de flúor al $5 \%$ (Duraphat ${ }^{\circledR}$ ) usado individualmente, mostraron clínicamente valores mayores de remineralización comparado con el grupo control. No se encontró diferencia estadísticamente significativa, al comparar la remineralización de lesiones de manchas blancas medidas a través de fluorescencia láser utilizando dos agentes remineralizantes, el barniz de flúor al 5\% (Duraphat ${ }^{\circledR}$ ), la nano-hidroxiapatita (Nano $\mathrm{P}^{\circledR}$ ) y una combinación de ambos agentes a los 30 días de su aplicación. Conclusión: La combinación del barniz de flúor al 5\% (Duraphat ${ }^{\circledR}$ ) y la nano-hidroxiapatita (Nano $\mathrm{P}^{\circledR}$ ), y barniz de flúor al $5 \%$ (Duraphat $^{\circledR}$ ) usado individualmente, mostraron clínicamente un incremento en la remineralización de las lesiones de manchas blancas a los 30 días de aplicación.

Palabras clave: remineralización dental, fluoruros tópicos, hidroxiapatita, fluorescencia

\section{ABSTRACT}

Objective: Evaluate the remineralization of white spot lesions on human premolar

Citar como: Romero-González MA, Aguilar-Gálvez D. Comparación de tres agentes remineralizantes utilizados en lesiones de manchas blancas en premolares medidos con fluorescencia láser: un estudio in vitro. Rev Cient Odontol (Lima). 2019; 7 (1): 66-77.

División de Odontopediatría, Carrera de Estomatología, Facultad de Ciencias de la Salud, Universidad Científica del Sur. Lima, Perú. 
enamel by laser-induced fluorescence following the use of a $5 \%$ fluoride varnish (Duraphat $\AA$ ), nano-hydroxyapatite (Nano $P()$, and the combination of both agents 30 days after application. Method: The sample consisted of 40 premolars divided into 4 groups, (1) control (without agent): artificial saliva, (2) $5 \%$ fluoride

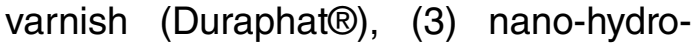
xyapatite (Nano $P(\mathbb{B})$, (4) and a combination of both remineralizing agents (nano-hydroxyapatite - Nano $\mathrm{P} \circledast$ and $5 \%$ fluoride varnish - Duraphat $\left.{ }^{\circledR}\right)$. The data were analyzed using the one-way ANOVA and Bonferroni tests. A $p$ value $<0.05$ was considered statistically significant. Results: Compared to the control group the highest remineralization values were obtained after the application of the $5 \%$ fluoride varnish (Duraphat $®$ ) and the nano-hydroxyapatite (Nano $P \AA)$, followed by the $5 \%$ fluoride varnish (Duraphat $\AA$ ) used individually. Conclusion: The combination of the $5 \%$ fluoride varnish (Duraphat $\circledast$ ) and the nano-hydroxyapatite (Nano $P \AA$ ), and the $5 \%$ fluoride varnish (Duraphat@) used individually improved remineralization of white spot lesions at 30 days.

Keywords: tooth remineralization, topical fluorides, durapatite, fluorescence

\section{INTRODUCCIÓN}

La caries dental es una enfermedad que provoca la destrucción del tejido dental debido a la presencia de ácidos orgánicos que son producidos por las bacterias cariogénicas situadas en el biofilm dental, sumado a un desequilibrio en el proceso de remineralización y desmineralización a través del tiempo $\left({ }^{1-4}\right)$. La mancha blanca representa clínicamente la primera observación de la caries dental, en la cual se evidencia la desmineralización de la subsuperficie del esmalte con un incremento de porosidad, ocasionado por la pérdida de minerales que se puede presentar tanto en la dentición temporal como en la permanente con la posibilidad de ser reversible en las primeras etapas $\left({ }^{5-9}\right)$, las cuales pueden ser además detectadas radiográficamente, con transiluminación o con modernos dispositivos de detección láser $\left({ }^{10}\right)$.

Las bacterias orales fermentan los carbohidratos y producen ácidos orgánicos que disminuyen el $\mathrm{pH}$ y por ende la disolución subsuperficial de los cristales de hidroxiapatita. En condiciones fisiológicas normales ( $\mathrm{pH} 7$ ), la saliva se encuentra supersaturada con iones de calcio y fosfato que se difunden en los espacios libres creados durante los ciclos de desmineralización $\left(^{2,3}\right)$.

Numerosos estudios se han enfocado en la prevención de la caries, promoviendo la remineralizaron de las manchas blancas utilizando agentes como el flúor, al ser un agente no invasivo muy comúnmente usado en el tratamiento de lesiones cariosas no cavitadas $\left({ }^{11}\right)$ dado que, su mecanismo de acción podría deberse a una mayor absorción del esmalte superficial y subsuperficial desmineralizado en comparación con el esmalte sano debido al aumento de la porosidad $\left({ }^{12,13}\right)$.

En un estudio previo, Carvalho et al. analizaron el efecto protector de los agentes remineralizantes sobre las manchas blancas. Llegando a la conclusión que la nano-hidroxiapatita mostró un efecto protector contra el desarrollo in vitro de manchas blancas comparado con el flúor barniz al $5 \%$.

La búsqueda de compuestos con beneficios adicionales y sinérgicos ha producido una plétora de agentes novedosos para la terapia de remineralización dirigida como la nano-hidroxiapatita que presenta un efecto desensibilizante como remineralizante $\left({ }^{14}\right)$; sin embargo, existe un número limitado de trabajos de 
investigación en los que se combina la aplicación de dos agentes con la finalidad de producir un efecto sinérgico y de esta forma repotenciar su acción como remineralizantes $\left({ }^{15}\right)$.

El uso de DIAGNOdent para evaluar la remineralización de las lesiones incipientes, después de la terapia preventiva, es una herramienta de monitoreo no invasiva confiable, pero se requieren más investigaciones para establecer firmemente la exactitud y fiabilidad de DIAGNOdent en la supervisión de la eficacia de la remineralización de varios métodos preventivos $\left({ }^{16}\right)$.

Por lo tanto, el propósito de este estudio fue evaluar la remineralización de lesiones de manchas blancas en el esmalte de premolares humanos a través de la fluorescencia láser utilizando el barniz de flúor al $5 \%\left(\right.$ Duraphat $\left.^{\circledR}\right)$, la nano-hidroxiapatita (Nano $\mathrm{P}^{\circledR}$ ) y la combinación de ambos agentes, 30 días después de su aplicación.

\section{MATERIALES Y MÉTODOS}

El tipo de investigación fue experimental, un estudio in vitro. El método de la investigación fue la observación estructurada. El universo de estudio estuvo conformado por 45 premolares, los cuales fueron extraídos debido a motivos ortodónticos y posteriormente donados para el estudio bajo consentimiento informado del paciente. Se excluyeron aquellos premolares que no cumplieron con los criterios de selección establecidos. La muestra estuvo conformada por 40 premolares, número que fue utilizado en hallazgos anteriores reportados por Hegde y Moany $\left({ }^{17}\right)$. La asignación de las piezas dentarias a los grupos se realizó en manera aleatoria. La prueba piloto se realizó con 4 muestras por cada grupo.
La muestra estuvo dividida en 4 grupos:

Grupo A: saliva artificial $(n=10)$

Grupo B: barniz de flúor al 5\% - Duraphat ${ }^{\circledR}$ (Colgate-Palmolive, Perú; Lote 011604).

Barniz con $5 \%$ de fluoruro de sodio, utilizado en el tratamiento de hipersensibilidad dental y prevención de caries $(n=10)$

Grupo C: nano-hidroxiapatita - Nano $\mathrm{P}^{\circledR}$ (FGM, Brasil; Lote 270417).

Agente desensibilizante y remineralizante basado en la tecnología de fosfato de calcio nanoestructurado $(n=10)$.

Grupo D: nano-hidroxiapatita - Nano $\mathrm{P}^{\circledR}$ (FGM, Brasil; Lote 270417) y barniz de flúor al 5\% - Duraphat ${ }^{\circledR}$ (Colgate-Palmolive, Perú; Lote 011604) $(n=10)$

\section{CRITERIOS DE SELECCIÓN}

\section{Criterios de inclusión}

- Premolares sanos (sin caries visible).

- Premolares superiores e inferiores.

- Premolares unirradiculares y birradiculares.

- Premolares extraídos debido a motivos ortodónticos, los cuales fueron posteriormente donados para el estudio.

- Premolares almacenados en solución isotónica salina por un tiempo no mayor a 10 meses.

\section{Criterios de exclusión}

- Premolares con lesiones de manchas blancas.

- Premolares con restauraciones y/o endodoncias.

- Premolares con abrasiones, fisuras o fracturas del esmalte. 
- Premolares que presentan defectos del desarrollo con afectación en la estructura (hipoplasias, hipocalcificaciones, hipomineralizaciones).

\section{Preparación de la muestra}

Se realizó profilaxis a los premolares con escobilla dental Robinson y agua destilada. Posteriormente, la muestra fue almacenada en solución isotónica salina, hasta que se realizó el procedimiento experimental. Se utilizó un molde estandarizado de material plástico y de forma cilíndrica; las muestras se colocaron de manera vertical en acrílico de autocurado. Se realizó un cuadrado de cinta adhesiva de $3 \mathrm{~mm} \times 3 \mathrm{~mm}$, el cual fue colocado en la superficie vestibular del esmalte sano y se pintó con barniz de uñas resistente al ácido (Revlon ColorStay Gel Envy ${ }^{\mathrm{TM}}$ Longwear Nail Enamel y ColorStay Gel Envy Diamond Top Coat ${ }^{\mathrm{TM}}$ ) toda la porción remanente del diente. Luego de 4 horas que el barniz de uñas secó totalmente, se retiró la cinta adhesiva.

\section{ALEATORIZACIÓN}

Se utilizó el muestreo aleatorio simple, las muestras fueron divididas en cuatro grupos (A-D) y cada grupo estuvo conformado por diez muestras $(n=10)$. A cada muestra de cada grupo se le asignó una denotación numérica $\left({ }^{1-10}\right)$.

\section{DESMINERALIZACIÓN DE LA MUESTRA}

En el esmalte expuesto de los premolares se crearon lesiones cariosas artificiales con una solución desmineralizante que estuvo compuesta por los siguientes insumos: 1 I de agua destilada, 0,264 g de fosfato de sodio, $0,244 \mathrm{~g}$ de cloruro de calcio y 0,20 $\mathrm{ml}$ de ácido acético con un $\mathrm{pH}$ ajustado a 4,0. Cada premolar se sumergió totalmente en un recipiente estéril individual que contenía $60 \mathrm{ml}$ de la solución desmineralizante durante 109 horas.

\section{MEDICIONES POSDESMINERALIZACIÓN}

Después de realizar la desmineralización en la superficie expuesta de los premolares, se realizó la medición posdesmineralización. Las muestras se lavaron con agua destilada y se dejaron secar con el aire de la jeringa durante 10 segundos. Luego de 10 minutos, se realizaron en todas las muestras la medición de la desmineralización usando el DIAGNOdent pen 2190 (Kaltenbach y Voigt $\mathrm{GmbH}$ y Co. KG, Biberach, Alemania).

\section{ALMACENAMIENTO DE LAS PIEZAS DURANTE EL PROCEDIMIENTO}

Durante el procedimiento experimental, cada premolar fue almacenado en un envase estéril de vidrio con $60 \mathrm{ml}$ de saliva artificial, la cual fue cambiada semanalmente según antecedentes (Al-Khateeb et al., 2014) $\left({ }^{18}\right)$.

La saliva artificial estuvo compuesta por: 1 I de agua destilada, $0,113 \mathrm{~g}$ de cloruro de calcio, $0,108 \mathrm{~g}$ de fosfato de sodio y $11,175 \mathrm{~g}$ de cloruro de potasio con un $\mathrm{pH}$ ajustado a 7,0.

\section{CEGAMIENTO}

Se planificó una investigación doble ciego para prevenir que los resultados de la investigación puedan estar influidos por el sesgo del observador al registrar las mediciones del DIAGNOdent pen 2190 a la ficha de recolección de datos. 


\section{PREPARACIÓN DE LAS MUESTRAS PARA LA REMINERALIZACIÓN}

Grupo A: saliva artificial

Las muestras de grupo control fueron expuestas a la saliva artificial por 30 días.

Grupo B: barniz de flúor al 5\% (Duraphat ${ }^{\circledR}$, Colgate).

Se secó la superficie.

El barniz de flúor fue aplicado en las manchas blancas con un pincel.

Después de 3 minutos la muestra se almacenó en saliva artificial.

Este procedimiento se realizó una vez por semana durante 30 días.

Grupo C: nano-hidroxiapatita (Nano $\mathrm{P}^{\circledR}$, FGM).

Se secó la superficie.

La nano-hidroxiapatita fue aplicada en las manchas blancas con la ayuda de un microaplicador desechable y con una taza de goma se friccionó el producto en la superficie dental durante 10 segundos. Se dejó el excedente del producto en reposo en contacto con la superficie durante 5 minutos y, después, se eliminó el exceso con bolas de algodón humedecidas levemente con agua.

Después de 3 minutos, la muestra fue almacenada en saliva artificial.

Este procedimiento se realizó una vez por semana durante 30 días.

Grupo D: nano-hidroxiapatita y barniz de flúor (Nano $\mathrm{P}^{\circledR}$, FGM y Duraphat ${ }^{\circledR}$, Colgate).
Se limpió y secó la superficie.

La nano-hidroxiapatita fue aplicada en las manchas blancas con la ayuda de un micro aplicador desechable y con una taza de goma se friccionó el producto en la superficie dental durante 10 segundos. Se dejó el excedente del producto en reposo en contacto con la superficie durante 5 minutos y, después, se eliminó el exceso con bolas de algodón humedecidas levemente con agua.

Después de 5 minutos se colocó el flúor barniz en las manchas blancas con un pincel, luego de 3 minutos la muestra fue almacenada en saliva artificial.

Este procedimiento se realizó una vez por semana durante 30 días.

Todos los agentes remineralizantes se aplicaron en un volumen de $0,03 \mathrm{ml}$ por muestra, lo que fue medido usando una jeringa de tuberculina.

\section{EVALUACIÓN DE LOS PARÁMETROS DE PRUEBA}

La duración del estudio experimental in vitro fue de 34 días y se realizaron 2 mediciones, la medición posdesmineralización (M1), y la medición en el día 30 de la aplicación de los agentes remineralizantes (M2). Las mediciones fueron realizadas utilizando el DIAGNOdent pen 2190, cada muestra antes de la respectiva medición se secó con aire de la jeringa triple durante 10 segundos debido a que la reproducibilidad del dispositivo aumenta en condiciones de sequedad, según antecedentes (Pinelli et al., 2010) $\left({ }^{19}\right)$.

\section{RECOLECCIÓN DE DATOS}

Los datos fueron recogidos en una ficha de recolección de datos para posteriormente ser analizados. 


\section{ANÁLISIS ESTADÍSTICO}

El análisis estadístico se realizó con ayuda del programa estadístico IBM SPSS Statistics para Windows versión 22.0 (Armonk, Nueva York).

Se encontró normalidad, se usó la prueba deAnova y el test de Bonferroni.Se trabajó en un nivel de significancia de 0,05.

\section{CONSIDERACIONES ÉTICAS}

Este estudio no presenta implicaciones éticas debido a que se trabajó en base a una muestra de 40 premolares, los cuales fueron donados luego de ser extraídos por motivos ortodónticos.

\section{RESULTADOS}

Al evaluar los valores de las mediciones de las lesiones de manchas blancas en los grupos de estudio a los 30 días se observó que la combinación de ambos agentes, el barniz de flúor al $5 \%$ (Duraphat ${ }^{\circledR}$ ), la nano-hidroxiapatita (Nano $\mathrm{P}^{\circledR}$ ), seguido del barniz de flúor al 5\%, mostraron clínicamente valores mayores de remineralización en comparación con el grupo control (tabla 1).

Al comparar la remineralización de lesiones de manchas blancas en esmalte de premolares humanos medidos a través de fluorescencia láser utilizando dos agentes remineralizantes, el barniz de flúor al $5 \%$ (Duraphat ${ }^{\circledR}$ ), la nanohidroxiapatita (Nano $\mathrm{P}^{\circledR}$ ) y una combinación de ambos agentes a los 30 días de su aplicación, no se encontró diferencia estadísticamente significativa (tabla 2).

Tabla 1. EVAluación de los VALORES de LAS MEdiciones dE LAS LESIONES DE MANCHAS BLANCAS EN LOS TRES GRUPOS DE ESTUDIO A LOS 30 DÍAS

\begin{tabular}{|c|c|c|c|c|c|}
\hline Grupos de estudio & Momentos & Media & $\begin{array}{l}\text { Desviación } \\
\text { estándar }\end{array}$ & Mínimo & Máximo \\
\hline \multirow[t]{2}{*}{ Saliva artificial } & Medición de desmineralización & 8,30 & 3,683 & 5 & 18 \\
\hline & $\begin{array}{l}\text { Medición de remineralización } \\
\text { (30 días) }\end{array}$ & 10,60 & 8,695 & 4 & 27 \\
\hline \multirow{2}{*}{$\begin{array}{l}\text { Barniz de flúor - } \\
\text { Duraphat }\end{array}$} & Medición de desmineralización & 6,60 & 2,319 & 3 & 10 \\
\hline & $\begin{array}{l}\text { Medición de remineralización } \\
\text { (30 días) }\end{array}$ & 6,50 & 1,841 & 5 & 11 \\
\hline \multirow{2}{*}{$\begin{array}{l}\text { Nano - hidroxiapatita } \\
\text { - Nano P }\end{array}$} & Medición de desmineralización & 7,50 & 2,173 & 5 & 12 \\
\hline & $\begin{array}{l}\text { Medición de remineralización } \\
\text { (30 días) }\end{array}$ & 7,50 & 5,397 & 3 & 22 \\
\hline \multirow{2}{*}{$\begin{array}{l}\text { Nano - hidroxiapatita } \\
\text { - Nano P y barniz de } \\
\text { flúor al } 5 \% \text { - Duraphat }\end{array}$} & Medición de desmineralización & 8,30 & 4,809 & 4 & 21 \\
\hline & $\begin{array}{l}\text { Medición de remineralización } \\
\text { (30 días) }\end{array}$ & 8,10 & 4,677 & 5 & 21 \\
\hline
\end{tabular}




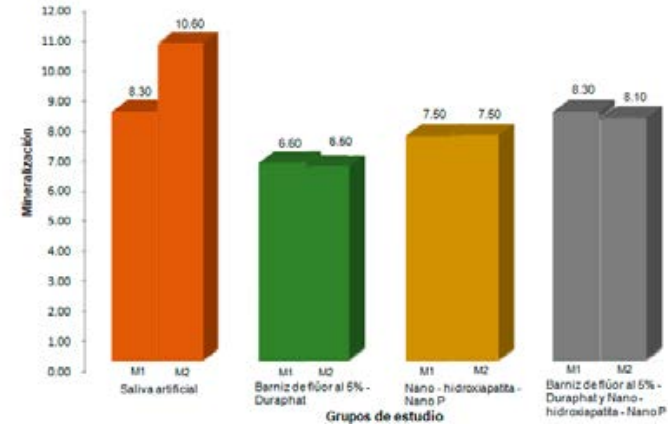

Gráfico 1. Evaluación de Los VALORES de LAS MEDICIONES DE LAS LESIONES DE MANCHAS BLANCAS EN LOS TRES GRUPOS DE ESTUDIO A LOS 30 DÍAS

TABLA 2. COMPARACIÓN DE LOS VALORES DE LA REMINERALIZACIÓN EN LOS TRES GRUPOS DE ESTUDIO EVALUADOS A LOS 30 DÍAS

\begin{tabular}{|l|l|l|l|l|c|}
\hline Grupo de estudio & Momento 1 & Momento 2 & $\begin{array}{c}\text { Valor } \\
p\end{array}$ & $\begin{array}{l}\text { Límite } \\
\text { infeior }\end{array}$ & $\begin{array}{c}\text { Límite } \\
\text { superior }\end{array}$ \\
\hline Saliva Artificial & $\begin{array}{l}\text { Medición de } \\
\text { desmineralización }\end{array}$ & $\begin{array}{l}\text { Medición de } \\
\text { remineralización } \\
\text { (30 días) }\end{array}$ & 0,908 & $-8,469$ & 3,869 \\
\hline Barniz de flúor al 5\% - Duraphat & $\begin{array}{l}\text { Medición de } \\
\text { desmineralización }\end{array}$ & $\begin{array}{l}\text { Medición de } \\
\text { remineralización } \\
\text { (30 días) }\end{array}$ & 1,000 & $-1,727$ & 1,927 \\
\hline Nano - hidroxiapatita - Nano P & $\begin{array}{l}\text { Medición de } \\
\text { desmineralización }\end{array}$ & $\begin{array}{l}\text { Medición de } \\
\text { remineralización } \\
\text { (30 días) }\end{array}$ & 1,000 & $-3,801$ & 3,401 \\
\hline Nano - hidroxiapatita - Nano P y & $\begin{array}{l}\text { Medición de } \\
\text { desmineralización }\end{array}$ & $\begin{array}{l}\text { Medición de } \\
\text { remineralización } \\
\text { (30 días) }\end{array}$ & 1,000 & $-1,637$ & 1,237 \\
\hline
\end{tabular}

Prueba de ANOVA de medidas repetidas (Test de Bonferroni).

\section{DISCUSIÓN}

La caries dental es un proceso dinámico que ocurre cuando la desmineralización excede a la remineralización $\left({ }^{20}\right)$. La caída en el $\mathrm{pH}$ de la saliva da como resultado la desmineralización, que si continúa conduce a la pérdida de minerales de la estructura del diente, lo que da como resultado la lesión de caries dental. Por otro lado, se puede producir una reversión si aumenta el pH y se produce una deposición de calcio, fosfato y flúor en la superficie dental $\left({ }^{20,21}\right)$.

Durante las primeras etapas de la caries dental, la intervención no invasiva puede convertir la lesión de mancha blanca en un estado inactivo $\left.{ }^{(22}\right)$, lo que resulta la mejor opción de tratamiento la aplicación de agentes remineralizantes $\left({ }^{23,24}\right)$. El propósito de este estudio fue evaluar la remineralización de lesiones de manchas blancas en el esmalte de premolares humanos a través de la fluorescencia láser utilizando el barniz de flúor al $5 \%$ (Duraphat $\left.{ }^{\circledR}\right)$, la nano-hidroxiapatita (Nano $\mathrm{P}^{\circledR}$ ) y la combinación de ambos agentes, 30 días después de su aplicación.

Se realizó un estudio experimental en el cual se trabajó con una muestra de 40 premolares superiores e inferiores. Se empleó el DIAGNOdent pen 2190, debido a que es una herramienta de 
monitoreo no invasiva confiable, para evaluar la remineralización de lesiones incipientes de las 40 muestras, las cuales fueron asignadas de manera aleatoria en 4 grupos. Para el desarrollo del estudio, se realizó previamente una prueba piloto con 4 muestras por cada grupo, en busca de perfeccionar la metodología. Por ese motivo, se decidió aumentar la cantidad de la solución desmineralizante y la saliva artificial de $40 \mathrm{ml} \mathrm{a} 60 \mathrm{ml}$ por recipiente, de modo que la muestra se encuentre totalmente sumergida.

Cabe resaltar que se realizó la capacitación correspondiente en el uso del DIAGNOdent pen 2190 con el gold estándar. Se planificó una investigación doble ciego para prevenir que los resultados de la investigación puedan estar influidos por el sesgo del observador al registrar las mediciones del DIAGNOdent pen 2190 a la ficha de recolección de datos.

El presente estudio demuestra que no existe diferencia estadísticamente significativa en la remineralización en los grupos de estudio; no obstante, se encontró clínicamente una mayor remineralización de las lesiones de manchas blancas utilizando un método combinado por dos agentes remineralizantes, el barniz de flúor al $5 \%$ (Duraphat ${ }^{\circledR}$ ) y la nano-hidroxiapatita (Nano $\mathrm{P}^{\circledR}$ ). Asimismo, el barniz de flúor al 5\% $\left(\right.$ Duraphat $^{\circledR}$ ) evidenció clínicamente un incremento en la remineralización de las superficies del esmalte dental comparado con la nanohidroxiapatita (Nano $\mathrm{P}^{\circledR}$ ), cuando fueron utilizados de manera individual. Estos resultados coincidieron con los encontrados por Chokshi et al. $\left({ }^{25}\right)$, quienes encontraron que el barniz de flúor al $5 \%$ presentó un incremento en la remineralización de la superficie del diente. Por otro lado, los resultados de Kamath et al. $\left({ }^{16}\right)$ discrepan de los encontrados en el estudio, debido a que reportaron que la remineralización del flúor barniz al $5 \%$ presentó un menor potencial de remineralización en comparación con la nano-hidroxiapatita (Nano $\mathrm{P}^{\circledR}$ ).

Aunque la remineralización entre la combinación de la nano-hidroxiapatita (Nano $\mathrm{P}^{\circledR}$ ) y el barniz de flúor al $5 \%$ (Duraphat ${ }^{\circledR}$ ) en el presente estudio resultó no presentar diferencia estadísticamente significativa, su utilidad clínica no debe subestimarse. En este estudio, el orden de aplicación fue la nano-hidroxiapatita $\left(\right.$ Nano $\mathrm{P}^{\circledR}$ ) seguido del barniz de flúor al $5 \%\left(\right.$ Duraphat $\left.{ }^{\circledR}\right)$. El mecanismo de acción de la nano-hidroxiapatita (Nano $\mathrm{P}^{\circledR}$ ) se basa en su capacidad de proveer iones de calcio, fosfato y fluoruro a la superficie desmineralizada del diente, que pueden reorganizarse en forma de hidroxiapatita, fluorapatita o fluoruro de calcio; sin embargo, su principal característica es proporcionar efecto desensibilizante, el cual se basa en la capacidad que la capa de hidroxiapatita tiene de ocluir los túbulos dentinarios, además del efecto de despolarización de fibras nerviosas debido al nitrato de potasio.

Teniendo en consideración que el mecanismo de acción del flúor barniz al 5\% (Duraphat ${ }^{\circledR}$ ) se basa en la capacidad de formación de fluoruro de calcio en la superficie del diente, lo que proporciona un depósito de fluoruro para la protección contra el ataque ácido. Además de este carácter de remineralización, el efecto desensibilizante se basa en la capacidad de formar glóbulos de fluoruro de calcio que ocluyen los túbulos dentinarios. Podrían ser estas las razones que explicarían un mayor grado de remineralización observado en este grupo cuando ambos agentes fueron usados conjuntamente $\left({ }^{25}\right)$.

Actualmente, no se conoce la interacción entre estos dos agentes remineralizantes, nano-hidroxiapatita (Nano $\mathrm{P}^{\circledR}$ ) y barniz de flúor al $5 \%$ (Duraphat ${ }^{\circledR}$ ), por lo que se planteó la aplicación de un 
método combinado con la finalidad de producir una mayor remineralización de las lesiones de mancha blanca que su uso individual.

El DIAGNOdent pen 2190 (Kavo, Biberach, Alemania) es un método no invasivo, que utiliza fluorescencia láser para medir la desmineralización temprana. Diversos estudios in vivo e in vitro han demostrado que el DIAGNOdent es un dispositivo para la detección de lesiones incipientes de esmalte en dientes permanentes $\left({ }^{14}\right)$. Al-Khateeb et al. $\left({ }^{26,27}\right)$, Eggertsson et al. $\left({ }^{28}\right)$, Mendes et al. $\left({ }^{29}\right)$, Pai et al. $\left({ }^{30}\right)$, Bahrololoomi et al. $\left({ }^{31}\right)$ y Patil et al. $\left({ }^{32}\right)$, en sus respectivos estudios también lo utilizaron para evaluar la eficacia de la remineralización de la superficie dental usando agentes remineralizantes. EI DIAGNOdent pen 2190 se utilizó en el presente estudio con el objetivo principal de comparar los cambios antes y después de la aplicación de los agentes remineralizantes.

No se encontró diferencia estadísticamente significativa comparando los grupos de estudio. Una de las limitaciones de este estudio fue el número de dientes utilizados. Además, hay que tener en cuenta que la remineralización in vitro es diferente a la remineralización in vivo, debido a que este último es un sistema complejo y dinámico. Por lo tanto, las extrapolaciones directas a las condiciones clínicas se deben realizar con precaución debido a las limitaciones obvias de los estudios in vitro, por lo que se requiere ensayos clínicos a largo plazo para establecer la utilidad de los agentes remineralizantes en las lesiones de manchas blancas en dientes en condiciones in vivo.
Se concluye que la combinación de ambos agentes, el barniz de flúor al $5 \%$ (Duraphat $^{\circledR}$ ) y la nano-hidroxiapatita (Nano $P^{\circledR}$ ), y el barniz de flúor al $5 \%$ usado individualmente, mostraron clínicamente un incremento en la remineralización de las lesiones de manchas blancas a los 30 días de aplicación.

\section{CONCLUSIONES}

1. La combinación de ambos agentes remineralizantes, el barniz de flúor al $5 \%\left(\right.$ Duraphat $\left.^{\circledR}\right)$ y la nano-hidroxiapatita (Nano $\mathrm{P}^{\circledR}$ ), y el barniz flúor al $5 \%$ (Duraphat ${ }^{\circledR}$ ) usado individualmente, mostraron clínicamente una mayor remineralización de las lesiones de manchas blancas a los 30 días de aplicación.

2. Los agentes remineralizantes usados en el estudio mostraron clínicamente un incremento en la remineralización de las superficies dentarias.

Contribución del autor: María Alejandra Romero Gonzales y Denisse Pilar Aguilar Gálvez han participado en la concepción del artículo, la recolección de información, su redacción y la aprobación de la versión final.

Fuente de financiamiento: Autofinanciado.

Conflicto de interés: Las autoras declaran no tener conflicto de interés de ningún tipo. 


\section{REFERENCIAS BIBLIOGRÁFICAS}

1. Struzycka I. The oral microbiome in dental caries. Pol J Microbiol. 2014; 63 (2): 127-35.

2. Robinson C, Shore RC, Brookes SJ, Strafford S, Wood SR, Kirkham J. The chemistry of enamel caries. Crit Rev Oral Biol Med. 2000; 11 (4): 481-95.

3. Dowker SEP, Anderson P, Elliott JC, Gao XJ. Crystal chemistry and dissolution of calcium phosphate in dental enamel. Mineralogical Magazine. 1990; 63 (6): 791-800.

4. Walsh T, Worthington HV, Glenny AM, Appelbe P, Marinho VC, Shi X. Fluoride toothpastes of different concentrations for preventing dental caries in children and adolescents. Cochrane Database Syst Rev. 2010; 20: CD007868.

5. Bergstrand F, Twetman S. A review on prevention and treatment of post-orthodontic white spot lesions-evidence-based methods and emerging technologies. Open Dent J. 2011; 5: 158-62.

6. Ferreira J, Soares M, Silva MFA, Oliveira AFB, Sampaio FC. Evaluation of different methods for monitoring incipient carious lesions in smooth surfaces under fluoride varnish therapy. Int J Paediatr Dent. 2008; 18 (4): 300-5.

7. Sudjalim T, Woods M, Manton D. Prevention of white spot lesions in orthodontic practice: a contemporary review. Aust Dent J. 2006; 51 (4): 284-9.

8. Torres C, Rosa P, Ferreira N, Borges AB. Effect of caries infiltration technique and fluoride therapy on microhardness of enamel carious lesions. Oper Dent. 2012; 37 (4): 363-9.

9. Lopatiene K, Borisovaite M, Lapenaite E. Prevention and treatment of white spot lesions during and after treatment with fixed orthodontic appliances: a systematic literature review. J Oral Maxillofac Res. 2016; 7 (2): 1-11.

10. Mount GJ. Defining, classifying, and placing incipient caries lesions in perspective. Dent Clin North Am. 2005; 49 (4): 701-23.

11. Walsh LJ. Contemporary technologies for remineralization therapies: A review. Int Dent S Afr. 2009; 4: 34-46.

12. White DJ. Reactivity of fluoride dentifrices with artificial caries. Effects on subsurface lesions: fluoride uptake, fluoride distribution, surface hardening and remineralisation. Caries Res. 1988; 22 (1): 27-36.

13. Itthagarun $\mathrm{A}$, Wei SH. Analysis of fluoride ion concentrations and in vitro fluoride uptake from different commercial dentifrices. Int Dent J. 1996; 46 (4): 357-61.

14. Kamath P, Nayak R, Kamath SU, Pai D. A comparative evaluation of the remineralization potential of three commercially available remineralizing agents on white spot lesions in primary teeth: An in vitro study. J Indian Soc Pedod Prev Dent. 2017; 35 (3): 229-37. 
15. Tange T, Sakurai $Y$, Hirose M, Noro D, Igarashi S. The effect of xylitol and fluoride on remineralization for primary tooth enamel caries in vitro. Pediatric Dental Journal. 2004; 14 (1): 55-9.

16. Naidu S, Tandon S, Nayak R, Ratnanag PV, Prajapati D, Kamath N. Efficacy of concomitant therapy with fluoride and chlorhexidine varnish on remineralization of incipient lesions in young children. Int J Clin Pediatr Dent. 2016; 9 (4): 296-302.

17. Hegde MN, Moany A. Remineralization of enamel subsurface lesions with casein phosphopeptide-amorphous calcium phosphate: A quantitative energy dispersive $\mathrm{X}$-ray analysis using scanning electron microscopy: An in vitro study. J Conserv Dent. 2012; 15: 61-7.

18. Al-Khateeb SN, Tarazi SJ, Al Maaitah EF, Al-Batayneh OB, Abu Alhaija ES. Eur Arch Paediatr Dent. Does acid etching enhance remineralisation of arrested white spot lesions? 2014; 15 (6): 413-9.

19. Pinelli C, Loffredo Lde C, Serra MC. Effect of drying on the reproducibility of DIAGNOdent to detect caries-like lesions. Braz Dent J. 2010; 21: 405-10.

20. Rao A, Malhotra N. The role of remineralizing agents in dentistry: A review. Compend Contin Educ Dent. 2011; 32: 26-33.

21. Winston AE, Bhaskar SN. Caries prevention in 21st century. J Am Dent Assoc. 1989; 129: 1579-87.

22. Featherstone JD. Dental caries: A dynamic disease process. Aust Dent J. 2008; 53: 286-91.

23. Rao R, Jain A, Verma M, Langade D, Patil A. Comparative evaluation of remineralizing potential of Fluoride using three different remineralizing protocols: An in vitro study. J Conserv Dent. 2017; 20 (6): 463-6.

24. Pretty IA. Caries detection and diagnosis: Novel technologies. J Dent. 2006; 34: 727-39.

25. Chokshi K, Chokshi A, Konde S, Shetty SR, Chandra KN, Jana S y col. An in vitro Comparative Evaluation of Three Remineralizing Agents using Confocal Microscopy. J Clin Diagn Res. 2016; 10 (6): 39-42.

26. Al-Khateeb S, Angmar Mannson B, de Josselin de Jong E. In vitro quantification of changes in caries lesions in orthodontic patients. J Dent Res. 1996; 75: 127.

27. Al-Khateeb S, Forsberg CM, de Josselin de Jong E, Angmar-Månsson B. A longitudinal laser fluorescence study of white spot lesions in orthodontic patients. Am J Orthod Dentofacial Orthop. 1998; 113: 595-602.

28. Eggertsson $\mathrm{H}$, Analoui M, van der Veen M, González-Cabezas C, Eckert G, Stookey G. Detection of early interproximal caries in vitro using laser fluorescence, dye-enhanced laser fluorescence and direct visual examination. Caries Res. 1999; 33: 227-33.

29. Mendes FM, Pinheiro SL, Bengtson AL. Effect of alteration in organic material of the occlusal caries on DIAGNOdent readings. Braz Oral Res. 2004; 18: 141-4. 
30. Pai D, Bhat SS, Taranath A, Sargod S, Pai VM. Use of laser fluorescence and scanning electron microscope to evaluate remineralization of incipient enamel lesions remineralized by topical application of casein phospho peptide amorphous calcium phosphate (CPP-aCP) containing cream. J Clin Pediatr Dent. 2008; 32: 201-6.

31. Bahrololoomi Z, Musavi SA, Kabudan M. In vitro evaluation of the efficacy of laser fluorescence (DIAGNOdent) to detect demineralization and remineralization of smooth enamel lesions. J Conserv Dent. 2013; 16: 362-6.

32. Patil N, Choudhari S, Kulkarni S, Joshi SR. Comparative evaluation of remineralizing potential of three agents on artificially demineralized human enamel: An in vitro study. J Conserv Dent. 2013; 16: 116-20. 